\title{
Intelligent Plant Disease Identification System Using Machine Learning ${ }^{\dagger}$
}

\author{
Paramasivam Alagumariappan 1,*, Najumnissa Jamal D 1, N M Gughan ${ }^{1}$, Bhaskar K B ${ }^{2}$, \\ Ramzan Ali Arshad Bilal ${ }^{1}$ and Vijayalakshmi $S^{3}$
}

1 Department of Electrical and Electronics Engineering, B. S. Abdur Rahman Crescent Institute of Science and Technology, Chennai 600048, India; najumnissa.d@crescent.education (N.J.D.); n.m.gughan@gmail.com (N.M.G.); warshath@gmail.com (R.A.A.B.)

2 Department of Electrical and Electronics Engineering, Adhi College of Engineering and Technology, Chennai 631605, India; jaibhaskar15@gmail.com

3 Department of Electronics and Communication Engineering, Vel Tech Rangarajan Dr. Sagunthala R\&D Institute of Science and Technology, Chennai 600062, India; sankarviji7@gmail.com

* Correspondence: paramasivam.eee@crescent.education; Tel.: +91-984-378-0801

+ Presented at the 7th International Electronic Conference on Sensors and Applications, 15-30 November 2020; Available online: https://ecsa-7.sciforum.net/.

Published: 15 November 2020

\begin{abstract}
Agriculture is the backbone of each and every country in the world. In India, most of the rural population still depends on agriculture. The agricultural sector provides major employment in rural areas. Further, it contributes a significant amount to India's Gross Domestic Product (GDP). So, protecting and enhancing the agricultural sector helps in developing India's economy. In this work, a real-time decision support system integrated with camera sensor module is designed and developed for identification of plant disease. Further, the performance of three machine learning algorithm such as Extreme Learning Machine (ELM), Support Vector Machine (SVM) with linear and polynomial kernels is analyzed. Results demonstrate that the performance of extreme learning machine is better when compared to the adopted support vector machine classifier. Also, it is observed that the sensitivity of support vector machine with polynomial kernel is better when compared to the other classifiers. This work appears to be of high social relevance since the developed real-time hardware is capable of detecting different plant diseases.
\end{abstract}

Keywords: plant disease; healthy; diseased; feature extraction; machine learning algorithm

\section{Introduction}

Plants are considered as a major resource for oxygen supply whereas they intake carbon dioxide and releases oxygen during day time for the photosynthesis process [1]. Plants are considered as the major supplier for food to all terrestrial organisms, including Human Beings. Plants not only provide food, but also other products like wood, oil, timber, fiber, medicine, dyes, etc. Plants transport an enormous amount of water from the soil to the atmosphere through transpiration [2].

In recent years, due to the rise in Global warming and deforestation, many plant species are on the verge of extinction. Also, the rise in pollution and industrialization decreased the plant population. Along with this, plant diseases also play a major role in their extinction. Anthracnose is a type of disease that develops dark, water-soaked lesions on stems, leaves, or fruit [3].

Plant diseases depend on Climatic conditions too. For instance, Reddish-purple leaf spots on the older leaves of the Ixora plant is due to entomosporium leaf spot fungus. Further, this disease is very dangerous which has active growth in cool and wet weather conditions. The various other 
diseases include Rust, Kole Roga, Yellow leaf disease, Leaf Rot, Leaf Curl, Angular Leaf Spot, Leaf Spot, Late Blight, Bacteria Wilt etc. affects the growth of the plants [4].

Nowadays several researchers have developed automatic recognition and identification algorithms for the classification of diseased plants [5-8]. Singh et al. (2019) [3] have developed an automated model for the identification of plant leaves diseases by implementing Computer Vision (CV), Machine Learning (ML), and Artificial Intelligence (AI). Further, the authors have trained their program code with 1070 images of Mango tree leaves with the help of TensorFlow.

Jaisakthi et al. (2019) [9] have proposed an automated identification system for detecting grapevine disease using image processing and machine learning algorithms such as Support Vector Machine (SVM), Random Forest and AdaBoost classifiers. Further, the authors have segmented leaves as healthy, black-rot, esca and leaf blight. Also, the authors have concluded that the overall accuracy of SVM is higher compared to Random Forest and AdaBoost classifiers.

Maniyath et al. (2018) [10] have utilized Random Forest classifier for classification of healthy and diseased leaves from the data set and incorporated Histogram of an Oriented Gradient (HOG) for extracting features of an image. Further, the authors have compared Random Forest classifier with various machine learning models such as Logistic regression, Support vector machine etc.

The objective of this work is to design and develop real-time decision sup-port hardware for the identification of healthy leaf and diseased leaf and to analyze the performance of the adopted machine learning classifiers such as extreme learning machine and support vector machine with linear and polynomial kernels.

\section{Materials and Methods}

\subsection{Proposed System}

Figure 1 shows the overall block diagram of the proposed system. In this work, a compact decision support system is designed and developed using Raspberry PI (System onboard hardware) to classify different plants, plants with and without diseases.

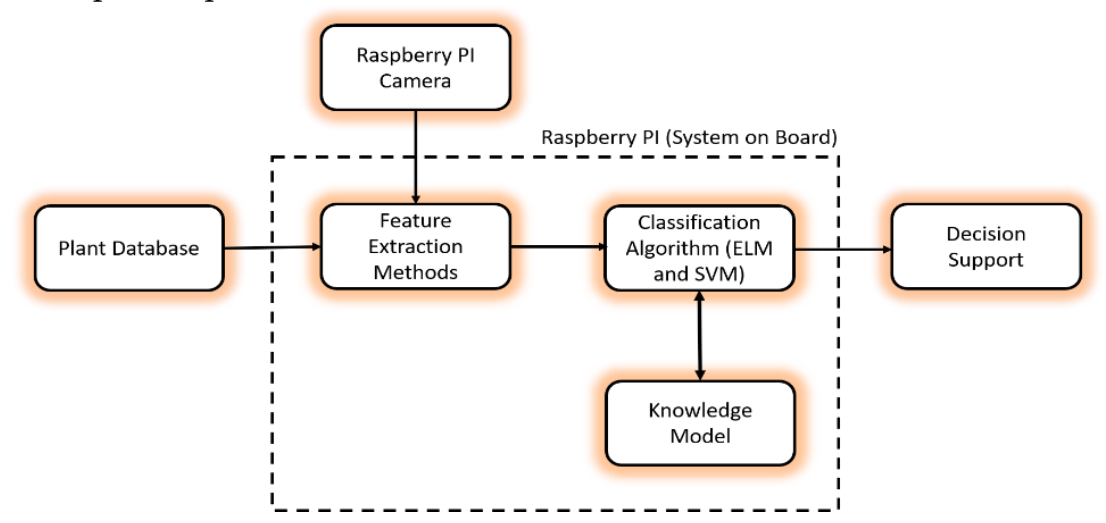

Figure 1. Overall block diagram of a real-time decision support system.

The plant data for different plants with and without diseases have been utilized to train the classifier model [11]. During the training phase, the plant images from the database are taken as input and the informative features using Hu moments and Haralick texture features are extracted from the image. Further, the extracted features are given to the two different classification algorithms such as Extreme Learning Machine (ELM) and Support Vector Machine (SVM) with Linear and Polynomial kernel (order $=8$ ). Also, the knowledge model is created at the end of the training phase. During the testing phase, the real-time plant image is acquired using Rasp-berry PI camera and is given to the feature extraction methods. Further, the classification algorithm classifies the plant with and without different diseases using previously acquired knowledge which acts as a decision support to the user.

\subsection{Extraction of Informative Features}


Hu moments: $\mathrm{Hu}$ moments also called $\mathrm{Hu}$ Moment Invariants consists of a set of 7 numbers that are determined using central moments that are invariant to image transformation. The initial 6 moments are invariant to rotation, reflection, translation, scale, while the seventh-moment sign varies with image transformation. Hu moments help to describe the outline of a particular leaf. Hu moments are determined over a single channel. The transformation consists of RGB to Grayscale calculations and subsequently to Hu moments [12].

Haralick texture: Haralick texture quantifies the texture of the leaf. Normally, the texture of healthy leaves and diseased leaves varies. To distinguish between the two, Haralick texture is used. Texture feature calculations use the contents of Gray Level Co-occurrence Matrix (GLCM) to give a measure of variation in intensity at the pixel of interest. GLCM is a square matrix and can be calculated at any angle (direction) and at any offset [13].

\subsection{Machine Learning Algorithms}

In this work, two different machine learning algorithms namely Support Vector Ma-chine and Extreme Learning Machine are utilized as a classifier to classify healthy and diseased leaves. Further, the SVM with two different kernels namely Linear and Polynomial kernel is adopted.

Support Vector Machine Classifier: Support Vector Machine Classification (SVM) is a binary classifier which falls under machine learning. It is a supervised learning model that analyzes data for classification and regression analysis.

As the proposed research is conducted based on the two-class binary classification problem, the most efficient hyperplane is selected by the SVM classifier, which divides all input samples into two classes. The effective hyperplane is considered as a classifier line and the sample inputs which are nearest to the classification line are called support vector. The expression for the training set of the SVM classifier is [14,15]:

$$
X=\left\{\left(y^{1}, z^{1}\right),\left(y^{2}, z^{2}\right), \ldots \ldots,\left(x^{n}, y^{n}\right)\right\}, y \in S^{n}, z \in\{-1,1\}
$$

The classification line is derived as:

$$
X(n)=(w . n)+a
$$

where $z$ represents the class label of $X$ and $n$ represents the sample Vector represented to a high dimensional space. With the help of $\mathrm{w}$ and a parameters, the equation of classification line is obtained. Further, the SVM classifier has 4 general kernel functions such as Linear, Polynomial, Gaussian, Hyperbolic tangent kernel whereas in this work, the Linear and polynomial is adopted [16]:

Linear: It is used when the data is linearly separated.

$$
k(x, y)=x^{T} y
$$

Polynomial: It allows learning for non-linear model which requires all the training data to be normalized.

$$
k(x, y)=\left(\alpha x^{T} y\right)^{d}
$$

where $x, y$ are vectors in input space and the parameters required are slope $\alpha$ and polynomial degree $d$.

In this work, the SVM based supervised machine learning classifier with two different kernels are trained using 200 train images of 3 different kinds of leaves. After the training process, the test images from the Raspberry PI camera are utilized to analyze the performance of the selected classifiers. The two different performance parameters, namely accuracy, and sensitivity, are calculated for SVM classifier with two different kernels such as Linear, and Polynomial.

Extreme Learning Machine (ELM) Classifier: Extreme Learning Machine (ELM) is a novel training algorithm used to train single hidden layer feedforward neural network (SLFNN) [17]. The computational speed of the ELM is high when compared to other traditional learning algorithms. ELM is utilized in all classification and regression problems. The ELM is derived by Shi et al. (2013) [18] 


$$
\sum_{i=1}^{h} \alpha_{i} \zeta_{i}\left(y_{n}\right)=\sum_{i=1}^{h} \alpha_{i} \zeta_{i}\left(w_{i} y_{n}+\beta_{i}\right)=x_{n}, n=1,2,3, \ldots . ., N
$$

where the hidden nodes are referred to as $h$, the weight vector or connection link between the input node to the $i$ th hidden node is $w_{i}$, the weight vector or connection link between the $i$ th hidden node to the output node is $\alpha_{i}$, and the threshold for the $i$ th hidden node is $\beta$.

\section{Results and Discussion}

In this work, 200 training images of each three different leaves are given to machine learning algorithms. Figure 2 shows a typical three different plant leaves such as Nerium oleander, Ixora coccinea and Sweet-scented geranium.

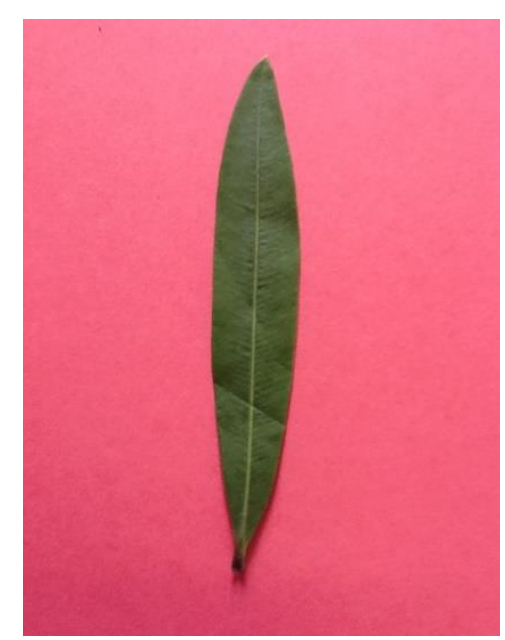

(a)

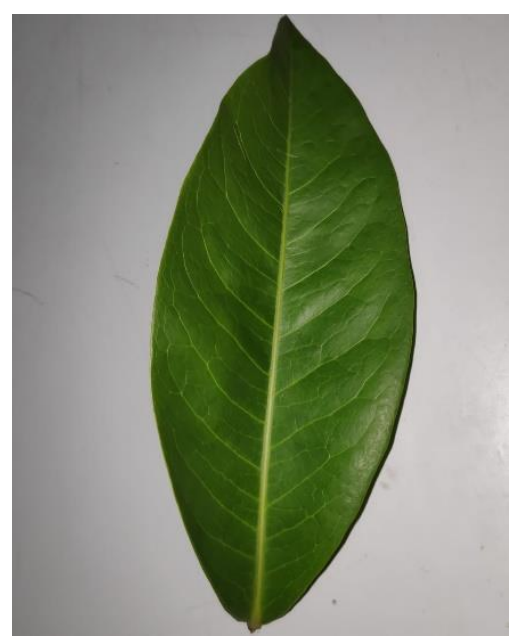

(b)

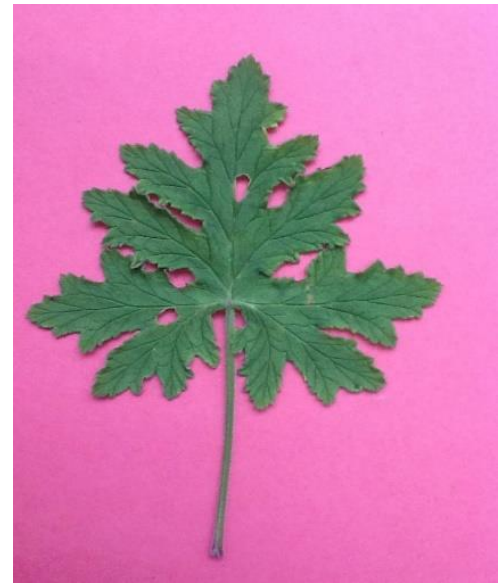

(c)

Figure 2. A typical plant leaves (a) Nerium oleander, (b) Ixora coccinea and (c) Sweet-scented geranium.

Figure 3 shows the accuracy of the three different classifiers such as SVM with Linear kernel (LSVM), SVM with Polynomial kernel (PSVM) and Extreme Learning Machine (ELM). Further, it is observed that the accuracy of extreme learning machine is higher when compared to the other two classifiers. 


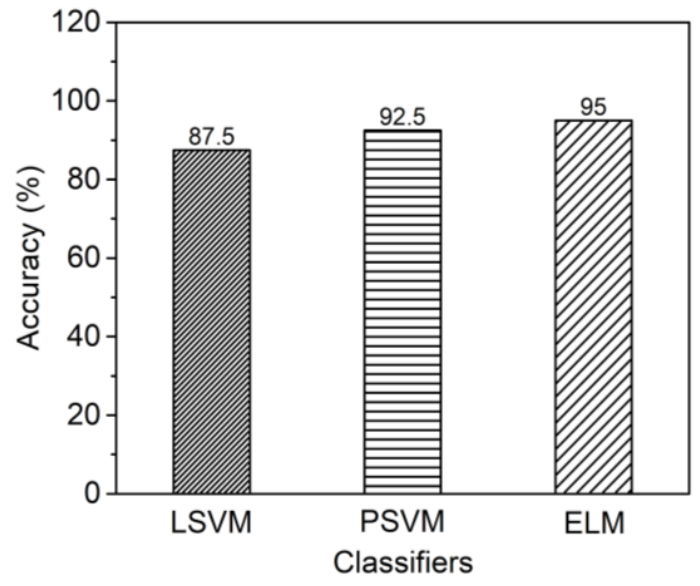

Figure 3. Accuracy of the different classifiers.

Figure 4 shows the sensitivity of the three different classifiers such as SVM with Linear kernel (LSVM), SVM with Polynomial kernel (PSVM) and Extreme Learning Machine (ELM). It is seen that the sensitivity of the extreme learning machine is higher when compared to the other two classifiers. Also, the sensitivity of PSVM is better and closer to the ELM classifier. Since the performance of extreme learning machine is better when compared to other two adopted classifiers, the ELM is utilized for decision support for real-time hardware. Figure 5 shows the decision support output of the proposed real-time hardware. The image of the real-time leaf is acquired using Raspberry PI camera and is given as a test image to the classification algorithm. Also, it is demonstrated that the proposed decision support system with ELM classifier is good at classifying healthy and diseased leaves in real-time.

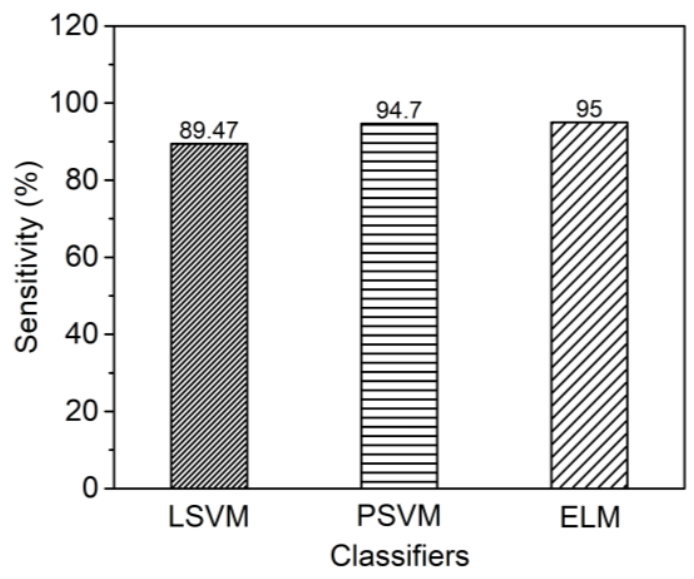

Figure 4. Sensitivity of the different classifiers. 


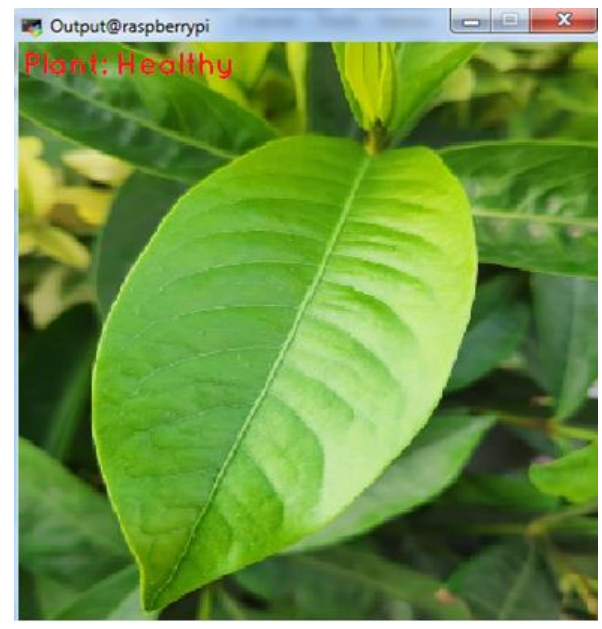

Figure 5. Decision support output of the proposed real-time hardware.

\section{Conclusions}

In this work, the performance of three machine learning algorithms such as extreme learning machine, support vector machine with linear and polynomial kernels were analyzed. Further, a realtime decision support system using extreme learning machine was designed and developed using Raspberry PI hardware. Results demonstrate that the performance parameters namely accuracy and sensitivity of extreme learning machine is $95 \%$ and is higher when compared to the other adopted classifiers. Also, it is observed that the developed real-time hardware with ELM classifier is highly capable of detecting three different plant diseases and can be extended to detect many more plant diseases by training it with wide range of train datasets.

Author Contributions: P.A. and N.J.D. conceived the idea of this manuscript. N.M.G. and R.A.A.B. designed the experiments and acquired the images. P.A., V.S. and B.K.B. have performed the analysis. All the authors have contributed towards preparing the manuscript. All the authors have read and approved the final manuscript. All authors have read and agreed to the published version of the manuscript.

Conflicts of Interest: The authors declare no conflict of interest.

\section{References}

1. Zhou, C.; Zhang, Z.; Yang, Y.; Gao, Q. Comparative analysis of photosynthesis of three Stipa species based on a photosynthesis model. In Proceedings of the 2011 IEEE Chinese Control and Decision Conference (CCDC), Mianyang, China, 23-25 May 2011; pp. 421-425.

2. Pipitsunthonsan, P.; Sopharat, J.; Sirisuk, P.; Chongcheawchamnan, M. Leaf Sensor for Stomata Transpiration Monitoring Using Temperature and Humidity. In Proceedings of the 2018 IEEE 21st International Symposium on Wireless Personal Multimedia Communications (WPMC), Chiang Rai, Thailand, 25-28 November 2018; pp. 252-255.

3. Singh, U.P.; Chouhan, S.S.; Jain, S.; Jain, S. Multilayer convolution neural network for the classification of mango leaves infected by anthracnose disease. IEEE Access 2019, 7, 43721-43729.

4. Raghavendra, B.K. Diseases Detection of Various Plant Leaf Using Image Processing Techniques: A Review. In Proceedings of the 2019 IEEE 5th International Conference on Advanced Computing \& Communication Systems (ICACCS), Coimbatore, India, 15-16 March, 2019; pp. 313-316.

5. Francis, J.; Anoop, B.K. Identification of leaf diseases in pepper plants using soft computing techniques. In Proceedings of the 2016 IEEE Conference on Emerging Devices and Smart Systems (ICEDSS), Namakkal, India, 4-5 March 2016; pp. 168-173.

6. Sabrol, H.; Satish, K. Tomato plant disease classification in digital images using classification tree. In Proceedings of the 2016 IEEE International Conference on Communication and Signal Processing (ICCSP), Melmaruvathur, India, 6-8 April 2016; pp. 1242-1246. 
7. Militante, S.V.; Gerardo, B.D.; Dionisio, N.V. Plant Leaf Detection and Disease Recognition using Deep Learning. In Proceedings of the 2019 IEEE Eurasia Conference on IOT, Communication and Engineering (ECICE), Yunlin, Taiwan, 3-6 October 2019; pp. 579-582.

8. Dhakate, M.; Ingole, A.B. Diagnosis of pomegranate plant diseases using neural network. In Proceedings of the 2015 IEEE Fifth National Conference on Computer Vision, Pattern Recognition, Image Processing and Graphics (NCVPRIPG), Patna, India, 16-19 December 2015; pp. 1-4.

9. Jaisakthi, S.M.; Mirunalini, P.; Thenmozhi, D. Grape Leaf Disease Identification using Machine Learning Techniques. In Proceedings of the 2019 IEEE International Conference on Computational Intelligence in Data Science (ICCIDS), Chennai, India, 21-23 February 2019, pp. 1-6.

10. Maniyath, S.R.; Vinod, P.V.; Niveditha, M.; Pooja, R.; Shashank, N.; Hebbar, R. Plant disease detection using machine learning. In Proceedings of the 2018 IEEEInternational Conference on Design Innovations for 3Cs Compute Communicate Control (ICDI3C), Bangalore, India, 25-28 April 2018; pp. 41-45.

11. Das, A.; Bucksch, A.; Price, C.A.; Weitz, J.S. ClearedLeavesDB: An online database of cleared plant leaf images. Plant Methods 2014, 10, 8.

12. Hu, N.M.-K. Visual pattern recognition by moment invariants. IEEE Trans. Inf. Theory 1962, 8, $179-187$.

13. Haralick, R.M.; Shanmugam, K.; Dinstein, I. Textural Features for Image Classification. IEEE Trans. Syst. Man Cybern. 1973, 6, 610-621.

14. Alagumariappan, P.; Krishnamurthy, K. A Thermal Sensor-Based Decision Support System for the Identification of Roof Leaks and Cracks. Proceedings 2020, $42,7$.

15. Onay, A.; Onay, M.; Abul, O. Classification of nervous system withdrawn \& approved drugs with ToxPrint features via machine learning strategies. Comput. Methods Prog. Biomed. 2017, 142, 9-19.

16. Bucurica, M.; Dogaru, R.; Dogaru, I. A comparison of extreme learning machine and support vector machine classifiers. In Proceedings of the 2015 IEEE International Conference on Intelligent Computer Communication and Processing (ICCP), Cluj-Napoca, Romania, 3-5 September 2015; pp. 471-474.

17. Ding, S.; Zhao, H.; Zhang, Y.; Xu, X.; Nie, R. Extreme learning machine: Algorithm, theory and applications. Artif. Intell. Rev. 2015, 44, 103-115.

18. Shi, J.; Cai, Y.; Zhu, J.; Zhong, J.; Wang, F. SEMG-based hand motion recognition using cumulative residual entropy and extreme learning machine. Med Biol. Eng. Comput. 2013, 51, 417-427.

Publisher's Note: MDPI stays neutral with regard to jurisdictional claims in published maps and institutional affiliations.

(C) 2020 by the authors. Submitted for possible open access publication under the terms and conditions of the Creative Commons Attribution (CC BY) license (http://creativecommons.org/licenses/by/4.0/). 